\title{
TEICHMÜLLER INEQUALITIES WITHOUT COEFFICIENT NORMALIZATION
}

\author{
BY \\ ARTHUR E. OBROCK $\left({ }^{1}\right)$
}

\begin{abstract}
Teichmüller's relation between the coefficients of extremal schlicht functions and quadratic differentials is extended. The coefficient normalization hypothesis in his theorem is dropped with the result that the new coefficient relations become more complex. This completes the partial result in this direction which is contained in Jenkins' General Coefficient Theorem. A modification of the version of the length-area method used by Teichmüller and Jenkins is introduced in our proof.
\end{abstract}

1. Introduction. In the theory of schlicht functions there is a rather vague but heuristic statement known as Teichmüller's Principle [6]. The problem is to give precise formulations of it. The original explicit inequalities of Teichmüller are either contained in or derivable from the much more extensive formulation of this principle, Jenkins' General Coefficient Theorem [2]. In its present form precise formulations are given when (1) $d \zeta^{2}$ is a positive (meromorphic) quadratic differential with poles on a finite Riemann surface $R$ and (2) $h$ is an "admissible" analytic homeomorphism which maps its "admissible" open set $D \subset R$ into $R$. An admissible function $h$ leaves poles of $d \zeta^{2}$ in $D$ fixed and omits poles not in $D$ from its range. In addition, at a pole of order greater than 3 there is a rather severe coefficient normalization on $h$.

This troublesome coefficient normalization is perhaps the most obvious obstruction to application of this principle to high order coefficient problems such as the resolution of Bieberbach's Conjecture. In this regard, we have only been able to use the present form to prove in general that $\operatorname{Re} A_{n} \leqq n$, when $A_{k}$ is real for $k \leqq[n / 2],[4]$.

The main purpose of this paper is to present a method which can be used to formulate Teichmüller's Principle even in the complete absence of coefficient normalization. What this amounts to is a distillation and refinement of the lengtharea method used by Jenkins. The approach in this paper can also be applied in

Received by the editors June 26, 1969 and, in revised form, April 9, 1970.

AMS 1969 subject classifications. Primary 3042, 3043.

Key words and phrases. Schlicht functions, quadratic differentials coefficient normalization, length-area method, Teichmüller's Theorem, Jenkins' General Coefficient Theorem.

(1) I wish to thank the NSF for support (contract number 6P-06361) during the preparation of this paper. I am indebted to the referee for his numerous suggestions and technical corrections, one of which affected the main formula.

Copyright (C) 1971, American Mathematical Society 
more general situations than mentioned above. For example we may use this method to consider problems where the range of $h$ is in a surface $S$ other than $R$. However, to avoid the inherent complications of a more general situation, we shall restrict ourselves in this paper to one of the more classical cases considered by Teichmüller.

Although our results are extensions of Teichmüller's inequality, they differ in character from their predecessors in two ways. First, in each situation there is a parametrized family of sharp inequalities rather than only one. There is also a wider but entirely analogous class of extremal functions. Second, in the nonnormalized case our coefficient relations are in general hyperelliptic in essence rather than algebraic as before.

With the exception of the general theory of geodesics of quadratic differentials on finite Riemann surfaces, this paper is intended to be elementary and selfcontained. The facts of this theory pertinent to our case are reviewed in $\$ 2$, the reader being referred to Jenkins [1, Chapter III], [3] for the proofs. Our format is to present the preparatory facts in $\S \S 3,4$. The core of the paper is then $\S 5$ where we describe the procedure in terms of Jenkins' development of the length-area method and state our main results. The proof and subsequent remarks are contained in the remaining sections.

2. Quadratic differentials. In this section we recall the well-known facts and terminology of quadratic differentials on finite Riemann surfaces, which will be used in this paper. Our standard reference is Jenkins' work [1, Chapter III], [3].

(a) Let $\{\pi=(z, U)\}$ denote a defining system of parameters $z: U \rightarrow C$ for a Riemann surface $R$. A quadratic differential $d \zeta^{2}$ assigns a meromorphic function $Q_{\pi}(z)$ on $z(U)$ to each parameter $\pi$, in such a way that the relation

$$
Q_{\sigma}(z)=Q_{\tau}(w(z))\left(w^{\prime}(z)\right)^{2}
$$

holds on $z(U \cap V)$ for any pair of parameters $\sigma=(z, U), \tau=(w, V)$. Here $w=w(z)$ denotes the conformal connecting map $w \circ z^{-1}: z(U \cap V) \rightarrow w(U \cap V)$, defined by the change in parameters on $R$. In view of this relation all information about $d \zeta^{2}$ is contained in $Q_{\pi}(z)$. Hence the notation $d \zeta^{2}=Q_{\pi}(z) d z^{2}$ can be used where $Q_{\pi}(z)$ is given explicitly.

If $z=z(p)$ is a parameter at $p_{0} \in R$ for which $z\left(p_{0}\right)=z_{0}$, then, near $z_{0}, Q_{\pi}(z)$ has the following Laurent expansion:

$$
Q_{\pi}(z)=\sum_{n=e}^{\infty} \alpha_{n}\left(z-z_{0}\right)^{n} \quad\left(\alpha_{e} \neq 0\right) .
$$

Although the coefficients $\alpha_{n}$ depend on the choice of $\pi$ the integer $e=e\left(p ; d \zeta^{2}\right)$ does not. $e$ is called the index of $d \zeta^{2}$ at $p ;|e|$ is called the order of $d \zeta^{2}$ at $p$. The following 
points and point sets may be defined in terms of the index $e$ :

$$
\begin{array}{ll}
Z=\{e>0\}, & \text { the zeros, } \\
P=\{e<0\}, & \text { the poles, } \\
A=\{e=0\}, & \text { the regular points, } \\
C=\{e \neq 0\}, & \text { the critical points, } \\
H=\{e \leqq-2\}, & \text { the infinite points, } \\
F=\{e \geqq-1\}, & \text { the finite points, } \\
G=\{e \geqq-1, e \neq 0\}, & \text { the finite critical points. }
\end{array}
$$

(b) The $Q$-metric $|d \zeta|$ and the $Q$-density $|d \zeta|^{2}$ are defined on $F$ in terms of the parameter $\pi$ by the equations $|d \zeta|=\left|Q_{\pi}(z)\right|^{1 / 2}|d z|,|d \zeta|^{2}=\left|Q_{\pi}(z)\right| d x d y$. The restriction of $|d \zeta|$ to $A$ is a conformal metric. If $F=A \cup Z$, in other words if $d \zeta^{2}$ has no simple poles, then $|d \zeta|$ is a complete Riemannian metric. This occurs in the case of interest of this paper. In the general case a $Q$-geodesic is an arc $\gamma$ with the "shortest join" property. At each point of $\gamma$ there is a neighborhood $N$ in which every subarc of $\gamma$ in $N$ is the shortest join of its end points among all arcs joining these points.

An isometry is a homeomorphism $h$ from a domain $D \subset F\left(d \zeta^{2}\right)$ into $F\left(d \omega^{2}\right)$ which preserves distance. Evidently $h$ also preserves geodesics. If $d \omega^{2}=d \zeta^{2}$ then $h$ is an autometry. The $Q$-integrals $\zeta=\int[Q(z)]^{1 / 2} d z$, on domains where they are conformal, are canonical examples of isometries into $F\left(d z^{2}\right)$, the complex plane. They are used to give a standard system of direction. For example, a horizontal (vertical) geodesic is a Jordan arc or curve which is mapped by $\zeta$ into a horizontal (vertical) line. A trajectory (orthogonal trajectory) is a maximal critical point free horizontal (vertical) geodesic. We say a trajectory $\gamma$ is unobstructed if (1) $\gamma$ is a Jordan curve or (2) $\zeta$ maps $\gamma$ onto the real line. A horizontal isometry preserves horizontal geodesics. (Note that $h$ is a horizontal isometry from $D \subset F\left(d \zeta^{2}\right)$ into $F\left(d \omega^{2}\right)$ if and only if $\omega \circ h \circ \zeta^{-1} \equiv \zeta+d$ locally on $\zeta\left[A\left(d \zeta^{2}\right) \cap h^{-1}\left(A\left(d \omega^{2}\right)\right)\right]$.) Finally, a translation is a horizontal autometry and a translation along trajectories sends subarcs of a trajectory $T$ in $D$ into $T$ itself.

(c) Given a piecewise smooth Jordan arc $\gamma=\gamma(t)$ in $F\left(d \zeta^{2}\right)$, we may continue $\zeta(z)$ analytically along both sides of $\gamma$ because $\zeta(z)$ is analytic inside and continuous on the closure of $U(t)$, a half neighborhood of $\gamma(t)$ for each $t$. Let us denote the sides of $\gamma$ (prime ends of $\gamma$ with respect to $R \backslash \gamma$ with the appropriate topology) by $\gamma_{r}, \gamma_{l}$. If $\zeta_{r}, \zeta_{l}$ are the analytic continuations of $\zeta$ along $\gamma_{r}, \gamma_{l}$ then, since $\zeta_{j}(\gamma(t \pm \varepsilon))$ $\neq \zeta_{j}(\gamma(t))$ for $\varepsilon$ small, we may define the right or left $\zeta$-angle at $\gamma(t)$ by the formula

$$
\lim _{\varepsilon \downarrow 0} \arg \left[\frac{\zeta_{j}(\gamma(t+\varepsilon))-\zeta_{j}(\gamma(t))}{\zeta_{j}(\gamma(t-\varepsilon))-\zeta_{j}(\gamma(t))}\right]=\psi_{j}(t)
$$

for $j=r$ or $l$ and arg, a continuous branch of the argument function. It is useful to describe geodesics in terms of the $\zeta$-angles. 
Lemma (2.3). A piecewise smooth Jordan arc $\gamma=\gamma(t)$ in $F$ is a geodesic of $d \zeta^{2}$ if and only if every $\zeta$-angle $\psi_{j}(t)$ on both sides of $\gamma$ satisfies $\psi_{j}(t) \geqq \pi$.

(2.4) A simple pole of $d \zeta^{2}(e=-1)$ is never the interior point of a geodesic.

(2.5) If $p$ is an interior point of a geodesic $\gamma$ of index $e \geqq 0$ then the two $\zeta$-angles at $p$ are $\theta$ and $\pi(e+2)-\theta$. Hence $\pi \leqq \theta \leqq(e+1) \pi$, and if $e=0, \theta=\pi$.

(2.6) If arg is a continuous branch of the argument function, $\gamma=\gamma(t)$ is a geodesic, and $\gamma(t) \in A$ for $t_{0}<t<t_{1}$, then $\arg \left[\zeta(\gamma(t))-\zeta\left(\gamma\left(t_{0}\right)\right)\right]$ is constant for $t_{0}<t<t_{1}$.

These remarks are similar to remarks in Jenkins' book [1, §3.2]. They follow from a study of the local behavior of $\zeta$.

(d) Trajectory structure on finite Riemann surfaces is defined in terms of regular curve families of horizontal geodesics. Local trajectory structure at $p$ is characterized by the index $e(p)$. Global trajectory structure is given in terms of a finite number of disjoint canonical domains which we call $Q$-domains. Let $K$ be a component of $\{p \in T: T$ is unobstructed $\}$. The interior of the closure of $K$ is a $Q$ domain.

In three exceptional cases the $Q$-domain is the whole surface $R$, which is either a sphere or a torus. There are five other types of $Q$-domains called end, strip, circle, ring and density domains. We recall the following facts concerning $Q$-domains for later use. For proofs, see $[1, \S \S 3.3,3.4]$.

LEMMA (2.7). End domains $E$ are bounded by a curve $\gamma$ containing one pole $p_{0}$ of order $|e| \geqq 3$ and at least one zero of $d \zeta^{2}$. The $\zeta$-integral maps $E$ conformally onto $\{ \pm \operatorname{Im} \zeta>c\}$. At $p_{0}$ there are $(|e|-2)$ end domains $E_{k}$ corresponding to the $(|e|-2)$ vertical directions $\theta_{k}+\pi /(|e|-2)$ where the horizontal directions

$$
\theta_{k}=-\left[\arg \alpha_{e}+2 \pi(k-1)\right] /(|e|-2) \quad(1 \leqq k \leqq|e|-2)
$$

are defined by the constant $\alpha_{e}$ of formula (2.1). The ends of the unobstructed trajectories, which sweep out $E_{k}$, tend to $p_{0}$ in the asymptotic directions $\theta_{k}$, $\theta_{l}$ respectively, where $l \equiv(k+1)(\bmod (|e|-2))$.

Lemma (2.8). Strip domains $S$ are bounded by two Jordan arcs $\gamma_{1}, \gamma_{2}$ each of which contains at least one finite critical point. The integral $\zeta$ maps $S$ conformally onto a strip $\left\{\left|\operatorname{Im}\left(\zeta-\zeta_{0}\right)\right|<w / 2\right\}$. The constant $w$ is called the "width" of $S$. The unobstructed trajectories, which sweep out $S$, tend to an infinite point $p_{1}$ in the direction $\theta\left(p_{1}\right)$ at the one end, to an infinite point $p_{2}$ in the direction $\theta\left(p_{2}\right)$ at the other end.

Lemma (2.9). The circle, ring and density domains are Jordan domains, whose closures contain closed geodesics of finite $|d \zeta|$-length.

Lemma (2.10). Boundary arcs of the $Q$-domains have $\zeta$-angles $\pi$ at every finite point on the side facing the Q-domain it bounds.

3. Teichmüller's case. (a) Teichmüller formulated his principle explicitly in several cases. We shall isolate one case and formulate the problem precisely there. 
Henceforth, the situation $\left(d \zeta^{2}, R ; h, D\right)$ described below will be called Teichmüller's case.

Let $m$ be a positive integer. On $R$ the Riemann sphere, let $d \zeta^{2}=Q(z) d z^{2}$ be a quadratic differential which has the following canonical form:

$$
Q(z)=\alpha_{m-1} z^{m-1}\left(1+\sum_{1}^{m-1} \beta_{v} z^{-v}\right),
$$

with $\alpha_{m-1} \neq 0$. Notice that $d \zeta^{2}$ is analytic on $R$ apart from a pole of order $(m+3)$ at $\infty$. An admissible domain $D$ with respect to $d \zeta^{2}$ is the complement in $R$ of a finite number of horizontal geodesics of finite length. (It will follow that in this case $D$ contains $\infty$ and is connected.) Living on this domain is the classical family $\Sigma(D)$ of schlicht functions $h$ which have the following normalized Laurent expansion at $\infty$ :

$$
h(z)=z+\sum_{n=1}^{\infty} c_{n} z^{-n}
$$

We may now state Teichmüller's Inequality.

THEOREM (TEICHMÜLLER). In Teichmüller's case if h has the coefficient normalization

$$
c_{n}=0 \text { for } 1 \leqq n \leqq m-1,
$$

then

$$
\operatorname{Re}\left\{\alpha_{m-1} c_{m}\right\} \leqq 0 .
$$

Equality occurs in (3.4) if and only if $f(z) \equiv z$.

(b) Our problem is to allow all schlicht functions on $D$ rather than those with condition (3.3). The inequalities (3.4) and the associated extremal functions, at which equality is attained, become more complicated. As a first step we note that a change of variables by a linear transformation will place the nonnormalized problem in Teichmüller's case. Second, we note that the General Coefficient Theorem [2] allows the relaxation of (3.3) to the weaker conditions:

$$
c_{n}=0 \text { for } 1 \leqq n \leqq[(m-2) / 2],
$$

where $[x]$ denotes the greatest integer in $x$. In this case, it is the translations of $D$ along trajectories, which are the extremal functions. Henceforth, (3.5) will be called Jenkins' normalization.

We should mention that Teichmüller's case is very closely related to the case studied by Schaeffer and Spencer in their book [5]. The only real difference is that their quadratic differentials may admit one simple pole. In that occurrence the trick used there of inducing a situation on a two sheeted covering surface of the sphere, branched at the two poles, will lead to Teichmüller's case with a pole of even order at $\infty$. In fact the induced quadratic differential $4 z^{2} Q\left(z^{2}\right) d z^{2}$ is even. 
(c) Certain properties are characteristic of Teichmüller's case. We shall review and develop the properties which will be used in our proof. We are especially concerned with the properties related to the asymptotic directions of trajectories at $\infty$. The first property concerns the uniqueness of trajectories in this case.

Lemma (3.6). In Teichmüller's case, any two points in $F=A \cup Z=R \mid\{\infty\}$ are joined by a unique geodesic $g\left(z_{1}, z_{2}\right)$.

Lemma (3.6) is Lemma XXI in [5]. It is also an immediate specialization of Jenkins' Lemma (4.4) in [1, p. 54] (since there is only 1 pole at $\infty$ in Teichmüller's case, the complement is simply connected).

Corollary (3.7). Admissible domains in Teichmüller's case are connected.

This follows from the Jordan curve theorem because (3.6) implies the absence of closed geodesics in this case. Referring to (2.9) we see that this fact also implies a simplified global trajectory structure, namely

COROllary (3.8). The Q-domains in Teichmüller's case consist of end and strip domains alone.

It is necessary that we examine the trajectory structure in Teichmüller's case in detail, paying close attention to the $m+1$ asymptotic directions of trajectories at $\infty$. Specifically these are

$$
\theta_{k}=\left[-\arg \left(\alpha_{m-1}\right)+2 \pi(k-1)\right] /(m+1),
$$

for $1 \leqq k \leqq m+1$ where $\alpha_{m-1} \neq 0$ is the leading coefficient in form (3.1) for $d \zeta^{2}$.

Lemma (3.10). In Teichmüller's case there are precisely $(m+1)$ end domains $E_{k}$ and at most $m-2$ strip domains $S_{j}$. Unobstructed trajectories in $E_{k}$ emanate from $\infty$ at angle $\theta_{k}$ and terminate at $\infty$ at angle $\theta_{l}$, where $l \equiv(k+1)(\bmod (m+1)) ;$ in $S_{j}$ they emanate from $\infty$ at angle $\theta_{k(j)}$ and terminate at $\infty$ at angle $\theta_{l(j)}$, where $k(j)+2$ $\leqq l(j) \bmod (m+1)$. The direction pairs $\left\{\left(\theta_{k}, \theta_{l}\right)\right\}$ of the $Q$-domains are distinct and do not separate one another.

Proof. We know from the general theory the asymptotic directions of trajectories at $\infty$ and the structure of the end domains $\left\{E_{k}\right\}_{1}^{m+1}$ there. We also know that the trajectories in one end of a strip domain tend to $\infty$ in the same direction.

Let $\left(\theta_{k}, \theta_{l}\right)$ be the directions of an unobstructed trajectory $T$. The uniqueness condition (3.6) implies $l \not \equiv k(\bmod (m+1))$, for in that event two points on the ends could also be joined by an orthogonal trajectory.

The disjointness of $Q$-domains and the Jordan curve theorem imply that pairs do not separate one another (i.e. $k_{1}<k_{2}<l_{1}<l_{2}$ ). This argument also shows that if $T_{1}, T_{2}$ have the same direction pairs $\left(\theta_{k}, \theta_{l}\right)$, then trajectories emanating between them in the $\theta_{k}$ direction terminate between them in the $\theta_{l}$ direction. Consequently if two $Q$-domains have the same directions we may find two which have boundary geodesics $\Gamma_{1}$ and $\Gamma_{2}$ which coincide at each end. Consequently $\Gamma_{1}-\Gamma_{2}$ would be by (2.10) a closed geodesic, which contradicts (3.6). 
We may view the $\theta_{k}$ as the vertices or 0 -simplexes of a regular $(m+1)$-polygon in the plane; the directions $\left(\theta_{k}, \theta_{l}\right)$ may be viewed as the sides or 1-simplexes. In such a geometric complex there can be at most $2 m-1$ sides. Since by (2.7) the $m+1$ sides $\left(\theta_{1}, \theta_{2}\right), \ldots,\left(\theta_{m}, \theta_{m+1}\right),\left(\theta_{m+1}, \theta_{1}\right)$ correspond to the end domains, there can be at most $m-2$ strip domains $S_{j}$, whose directions $\left(\theta_{k(j)}, \theta_{l(j)}\right)$ satisfy the conditions $l(j) \geqq(k(j)+2)(\bmod (m+1))$. Examples show that all possible geometric complexes which contain the sides of the regular polygon occur.

(d) The geodesic $g\left(z_{1}, z_{2}\right)$ which connects $z_{1}$ to $z_{2}$ is unique. In this section we define a canonical and rigid homotopy $F(t, s)=g_{s}(t)$ which deforms $g_{0}(t)$ into $g_{\theta}(t)$. We shall refer to it by saying we "bend the right (or left) end (of the geodesic $g$ ) $\theta$ radians toward the left (or right) side of $g$ ". In fact $F(t, s)=g_{s}(t)$ will be a geodesic for each fixed $s$ and one end of $g=g_{0}$ will be rigid $\left(F(t, s) \equiv F(t, 0)\right.$ for $t \geqq t_{0}$, $0 \leqq s \leqq \theta$ ). Of course left and right refers to an orientation of $g$ and radians are measured by the change in $\arg \left[\zeta\left(F\left(t_{1}, s\right)\right)-\zeta_{0}\right]$ as $s$ varies.

Let $g$ be a geodesic of finite or infinite length. Lemma (2.3) implies that all $\zeta$-angles on the left (or right) side of $g$ are at least $\pi$ radians. If all $\zeta$-angles on the left (right) side are equal to $\pi$ then we say that $g$ cannot be bent toward its left (right) side. If some point $z_{1}$ exists where the left $\zeta$-angle exceeds $\pi$ then by (2.5) $z_{1}$ must be a zero of $d \zeta^{2}$. We may assume that $z_{1}$ is the farthest point left of such points. We write $g=h_{0}+g_{1}$, where $h_{0}$ are the points of $g$ left of $z_{1}$ and $g_{1}$ are the points of $g$ right of $z_{1}$. By (2.6), $\arg \left(\zeta\left(h_{0}(t)\right)-\zeta\left(z_{1}\right)\right)$ is constant on int $h_{0}$. In fact $\zeta$ can be defined conformally on a neighborhood of int $h_{0}$ and $h_{0}=\zeta^{-1}\left(\zeta\left(z_{1}\right)+t e^{i \alpha}\right)$ for $t \geqq 0$. We then set

$$
h_{s}(t)=\zeta^{-1}\left(\zeta\left(z_{1}\right)+t e^{i(\alpha-s)}\right)
$$

for $t \geqq 0, s$ small, and define

$$
F(t, s)=g_{s}(t)=g_{s}=h_{s}+g_{1} .
$$

If this is defined for $0 \leqq s \leqq \varepsilon$ then $g_{\varepsilon}$ is the result of bending the left end of $g, \varepsilon$ radians toward the left. As $s$ increases, the left $\zeta$-angle at $z_{1}$ decreases. Therefore $g_{s}$ will be a geodesic by (2.3) as $s$ increases until either

(i) the $\zeta$-angle of $g_{s}$ on the left at $z_{1}$ equals $\pi$, or

(ii) int $h_{s}$ contains a zero of $d \zeta^{2}$.

In case (i) we search to the right of $z_{1}$ for the first zero whose left $\zeta$-angle exceeds $\pi$. If none can be found then all left angles are $\pi$ and $g_{s}$ cannot be bent farther toward the left. If one can be found, say $z_{2}$, then we may repeat the process from the pivot $z_{2}$, adding the new radians to the ones already accumulated.

If case (ii) occurs then we let $z_{2}$ be the farthest interior zero of int $h_{s}$, left on $h_{s}$. Since the right $\zeta$-angle at $z_{2}$ must be $\pi$, the left $\zeta$-angle must be $\left(e\left(z_{2}\right)+1\right) \pi$ and we may repeat the process using $z_{2}$ as the new pivot and add the new radians to the total radians accumulated already.

(e) Next we consider the half plane domains

$$
H_{k}=\left\{(-1)^{k-1} \operatorname{Re} \zeta>d_{k}\right\} \quad(1 \leqq k \leqq m+1)
$$


which correspond to the horizontal asymptotic direction $\theta_{k}$ of (3.9). Let $g$ be a geodesic with ends of infinite length with one end in $H_{k}$ and the other in $H_{l}$. We may assume that $k<l$ and that $g$ is sensed left to right as we run from $H_{l}$ to $H_{k}$. Consider the left side of $g$. If all its $\zeta$-angles are $\pi$ then there are vertical translates of $g$ which are unobstructed geodesics, namely $\zeta^{-1}(\zeta(g(t))+i s)(-\infty \leqq t \leqq \infty)$, $0 \leqq s<s_{1}$, and similarly on the right side for $0 \geqq s>s_{2}$. We suppose $s_{1}$ and $s_{2}$ are the largest and smallest such numbers. Clearly both $s_{1}=+\infty$ and $s_{2}=-\infty$ is only possible when $Q(z) d z^{2} \equiv \alpha_{m-1} d z^{2}$, in which case there are no proper end domains. Otherwise there are 3 possible cases:

(i) $\max \left(\left|s_{1}\right|,\left|s_{2}\right|\right)=\infty$,

(ii) $0<\max \left(\left|s_{1}\right|,\left|s_{2}\right|\right)<\infty$,

(iii) $0=\max \left(\left|s_{1}\right|,\left|s_{2}\right|\right)$.

In the first case (i) we say that $H_{k}, H_{l}$ are end connected. The translates of $g$ span a set $K$ on which $\zeta$ is defined conformally and maps onto a half plane $\left\{\operatorname{Im} e^{i \alpha} \zeta>c\right\}$. Since $d \zeta^{2}$ has only $(m+3)-4$ zeros this implies that there is a horizontal end domain $\{\operatorname{Im} \zeta>c\}$ which meets both $H_{k}$ and $H_{l}$. Hence by (2.7), $|k-l| \equiv$ $1 \bmod (m+1)$.

In the second case (ii) there is a strip $\left\{\left|\operatorname{Im} e^{i \alpha}\left(\zeta-\zeta_{0}\right)\right|<w / 2\right\}$ parallel to one side of $g$ which meets both $H_{k}$ and $H_{l}$. We call this pair $H_{k}, H_{l}$ strip connected. There are actually two subcases which are important:

(iia) $\left\{\left|\operatorname{Im}\left(\zeta-\zeta_{0}\right)\right|<w / 2\right\}$ meets $H_{k}$ and $H_{l}$,

(iib) not (iia),

according to whether or not a horizontal strip connects $H_{k}$ to $H_{l}$.

The remaining case (iii) is called line connected. No unobstructed geodesic meets both $H_{k}$ and $H_{l}$.

Next we define a closed set $D_{k l}$ for each pair, called the connecting domain. In the end connected case (i) we bend the ends of $\partial E$, the boundary of the connecting end domain $E$, back toward the vertical, that is, until the ends are vertical geodesics. The connecting domain $D_{k l}$ is the union of $E$ and the deformations of $\partial E$. In the strip connected case we bend the ends of $\partial S$, the boundary of a connecting strip $S$, away from $S$ toward the vertical. The connecting domain $D_{\kappa l}$ is the union of $S$ and these deformations of $\partial S$. Finally in the line connected case we may bend the ends of the connecting geodesic $g$ back toward the vertical. The connecting domain $D_{k l}$ is the union of these deformations of $g$.

In cases (i), (ii) it is possible to define a conformal branch of the $Q$-integral, say $\omega$, on int $D_{k l}$, because int $D_{k l}$ is simply connected and critical point free. The image $\omega\left(D_{k l}\right)$ is diagramed as the shaded area in (3.11) Figures 1, 2a, b. In case (iii), int $D_{k l}$ consists of 2 components both simply connected and joined by the connecting geodesic $g$. Here $\omega$ denotes a branch of the $Q$-integral defined conformally in one component and extended along one side of $g$ into the other. The case where the images of the components do not overlap is diagramed as the shaded area in (3.11) Figure 3. 
1. End connected

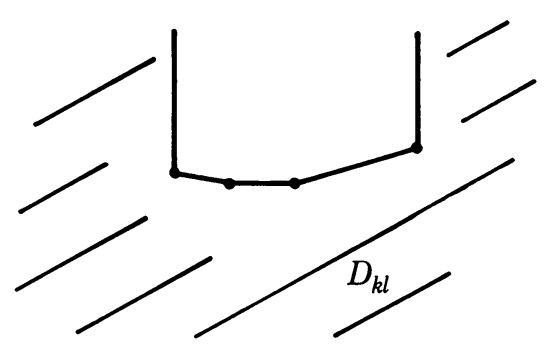

2a. Strip connected

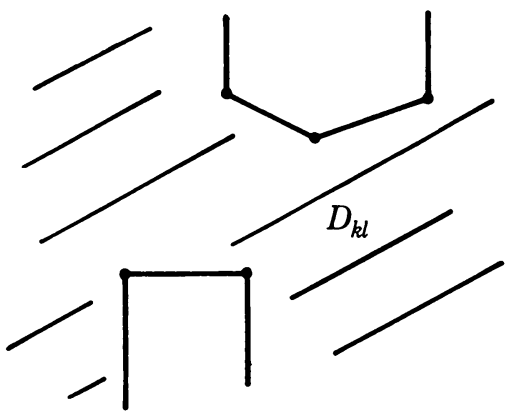

(3.11) 2b. Strip connected

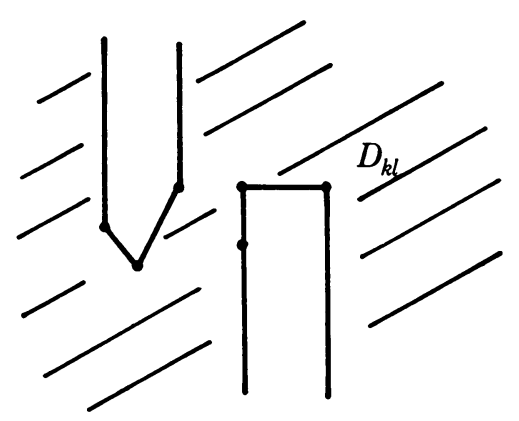

3. Line connected

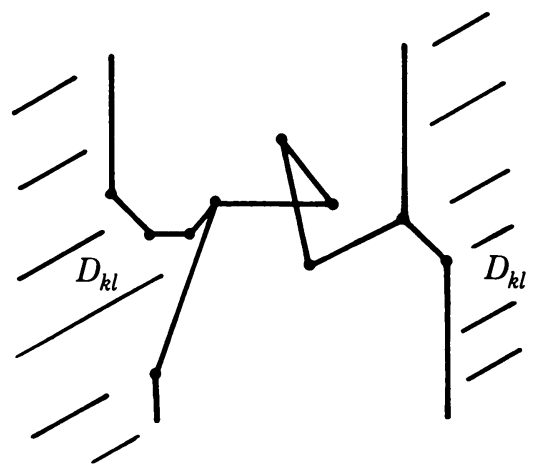

4. Horizontal direction adjustment constants. (a) We begin by studying $\zeta$ a fixed branch of the $Q$-integral. Let $V$ denote a fixed geodesic, say vertical, with precisely one end emanating from $\infty$. The local trajectory structure of $d \zeta^{2}$ at $\infty$ implies that, for $r \geqq r_{0}, U=\{|z|>r\}-V$ is simply connected and critical point free. Hence $\zeta=\int Q^{1 / 2} d z$ may be defined as a locally conformal analytic function on $U$.

End domains $E_{k}$ of $d \zeta^{2}$ and end domains $H_{k}$ of $-d \zeta^{2}$ for $1 \leqq k \leqq m+1$ will be called horizontal, vertical half plane domains respectively. We may assume $V$ lies in $E_{m+1}$. Then the restriction of the direct analytic continuation of $\zeta$ to these domains maps them conformally onto

$$
\begin{aligned}
& \zeta\left(H_{k}\right)=\left\{(-1)^{k+1} \operatorname{Re} \zeta>a_{k}\right\}, \\
& \zeta\left(E_{k}\right)=\left\{(-1)^{k+1} \operatorname{Im} \zeta>b_{k}\right\},
\end{aligned}
$$

for $1 \leqq k \leqq m+1$.

Henceforth we reserve $\zeta$ to refer to this fixed branch of the $Q$-integral. We shall use the fact that since $d \zeta^{2}$ is a quadratic differential, any other branch $\omega$ of the $Q$-integral is related to $\zeta$ by an equation of the form

$$
\omega= \pm \zeta+d .
$$

We call $d$ a quasi-period. 
We wish to expand $\zeta(z)$ in $U$. Hence we observe from the canonical form (3.1) for $Q(z)$ that

$$
\begin{aligned}
Q(z)^{1 / 2} & =\alpha_{m-1}^{1 / 2} z^{(m-1) / 2}\left[1+\sum_{v=1}^{m-1} \beta_{v} z^{-v}\right]^{1 / 2} \\
& =\alpha_{m-1}^{1 / 2} z^{(m-1) / 2}\left[1+\sum_{j=1}^{\infty}\left(\begin{array}{c}
1 / 2 \\
j
\end{array}\right)\left(\sum_{v=1}^{m-1} \beta_{v} z^{-v}\right)^{j}\right] \\
& =\alpha_{m-1}^{1 / 2} z^{(m-1) / 2}\left[1+\sum_{j=1}^{\infty}\left(\begin{array}{c}
1 / 2 \\
j
\end{array}\right) \sum_{S(j)}\left(\begin{array}{c}
j \\
k_{1}, \ldots, k_{m-1}
\end{array}\right) \prod_{v=1}^{m-1}\left(\beta_{v} z^{-v}\right)^{k_{v}}\right]
\end{aligned}
$$

where

$$
\begin{aligned}
S(j) & =\left\{k_{1}, \ldots, k_{m-1}: k_{v} \geqq 0, k_{1}+\cdots+k_{m-1}=j\right\}, \\
\left(\begin{array}{c}
1 / 2 \\
j
\end{array}\right) & =\frac{(1 / 2)(1 / 2-1) \cdots(1 / 2-j+1)}{j !}, \\
\left(\begin{array}{c}
j \\
k_{1}, \ldots, k_{m-1}
\end{array}\right) & =\frac{j !}{k_{1} ! k_{2} ! \cdots k_{m-1} !}
\end{aligned}
$$

Hence after collecting the coefficients belonging to the powers (possibly fractional) of $z$ and integrating term by term we obtain the following desired expansion:

$$
\zeta=\sum_{n \geqq 0}^{\prime} \sigma_{n} z^{(m+1) / 2-n}+\sigma_{l} \log z+\sigma_{c},
$$

where prime denotes the absence of a constant term (when $m+1$ is even), $\sigma_{l}=0$ when $m+1$ is odd, and $\sigma_{c}$ is the constant of integration. In addition we have a formula for the coefficients

$$
\sigma_{n}=\frac{2 \alpha^{1 / 2}}{(m+1-2 n)}\left[\sum_{I(n)}\left(\begin{array}{c}
1 / 2 \\
k_{1}, \ldots, k_{m-1}
\end{array}\right) \prod_{v=1}^{m-1} \beta_{v^{\nu}}^{k}\right],
$$

where

$$
\begin{aligned}
\left(\begin{array}{c}
1 / 2 \\
0,0, \ldots, 0
\end{array}\right) & =1, \\
I(n) & =\left\{\left(k_{1}, \ldots, k_{m-1}\right): k_{v} \geqq 0,1 k_{1}+2 k_{2}+\cdots+(m-1) k_{m-1}=n\right\}, \\
\left(\begin{array}{c}
1 / 2 \\
k_{1}, \ldots, k_{m-1}
\end{array}\right) & =\frac{(1 / 2)(1 / 2-1) \cdots\left(1 / 2-k_{1}-\cdots-k_{m-1}+1\right)}{k_{1} ! k_{2} ! \cdots k_{m-1} !} .
\end{aligned}
$$

Notice that $k_{v}=0$ for $\nu>n$ in $I(n)$. Also, this formula holds for $\sigma_{l}$ when $(m+1)$ is even, provided that we employ the conventions $l=n=(m+1) / 2$ and $2 /(m+1-2 l)$ $=1$ in that case.

From expansion (4.3) we note that if $z, e^{2 \pi i} z$ represent the prime ends of $U-V$ at $z \in V$, then

$$
\zeta\left(e^{2 \pi i} z\right)=e^{(m+1) \pi i}(\zeta(z)+q),
$$

where the quasi-period $q$ satisfies

$$
q=2 \pi i \sigma_{l}+\left(e^{(m+1) \pi i}-1\right) \sigma_{c} .
$$

In other words $q=2 \pi i \sigma_{l}$ when $m+1$ is even and $q=-2 \sigma_{c}$ when $m+1$ is odd. 
(b) Next we define some canonical parametrized exhaustions. Let $\gamma$ denote any Jordan curve on the sphere, not containing the point $\infty$. Then $U_{y}, J_{y}$ denote the Jordan domains bounded by $\gamma$ containing, not containing $\infty$, respectively. They are also of infinite, finite $|d \zeta|^{2}$-density respectively.

Recall now that $\zeta$ is defined on $U=\{|z|>r\}-V$, for a vertical geodesic $V$. Then let $\gamma_{1}=\gamma_{1}(r ; \zeta)=\{z \in U:|\zeta(z)|=r\}$ and let $\gamma_{2}=\gamma_{2}(r ; \zeta)$ equal the geodesic on $V$ which joins the end points of $\gamma_{1}$. Finally we define $\gamma_{r}=\gamma_{1}+\gamma_{2}$. Evidently we have

$$
\zeta\left(\gamma_{1}(\theta)\right)=r e^{i \theta}
$$

for $\theta_{1}(r) \leqq \theta \leqq \theta_{2}(r)$, where

$$
\begin{aligned}
& \theta_{1}(r)=-\frac{\pi}{2}+\sin ^{-1} \frac{a_{1}}{r} \\
& \theta_{2}(r)=-\frac{\pi}{2}+(m+1) \pi+e^{(m+1) \pi i} \sin ^{-1}\left(\frac{a_{1}+\operatorname{Re} q}{r}\right)
\end{aligned}
$$

provided $V$ is on the right boundary of $H_{1}$ (see (4.1)).

(c) The ends of all horizontal geodesics of infinite length tend to the point $\infty$ in one of the $m+1$ horizontal directions $\theta_{k}$ of (3.9) for $1 \leqq k \leqq m+1$. The direction $\theta_{k}$ corresponds to the vertical half plane $H_{k}$. Now $\gamma_{1}(r ; \zeta) \cap H_{k}$ contains a unique point $x_{k}=x_{k}(r, \zeta)$ for which $\zeta\left(x_{k}\right)=(-1)^{k-1} r$. We shall prove that

$$
\int_{g\left(x_{k}, x_{j}\right)}|d \zeta|=2 r+h_{j k}+o(1)
$$

The constants $h_{j k}=h_{j k}(\zeta)$ clearly depend on the branch $\zeta$ and will be called the horizontal direction adjustment constants (hdac) of $\zeta$. However, when $\zeta$ is fixed we shall suppress its dependence on $\zeta$ in our notation.

We require a slightly stronger statement than (4.9). Let $z_{k}$ denote an arbitrary point in $H_{k}$. Set

$$
z_{k}(t)=\zeta^{-1}\left(\zeta\left(z_{k}\right)+(-1)^{k-1} t\right)
$$

for $t \geqq 0,1 \leqq k \leqq m+1$. Then if $g(z, w)$ denotes the geodesic with end points $z$ and $w$ and $g(t)=g\left(z_{k}(t), z_{j}(t)\right)$, we prove

LEMMA (4.11). Under the preceding notation

$$
\begin{aligned}
& \int_{g\left(z_{k}(t), z_{k+1}(t)\right)}|d \zeta| \geqq(-1)^{k-1} \operatorname{Re} \zeta\left(z_{k}(t)\right)+(-1)^{k} \operatorname{Re} \zeta\left(z_{k+1}(t)\right), \\
& \int_{g\left(z_{1}(t), z_{m+1}(t)\right)}|d \zeta| \geqq \operatorname{Re} \zeta\left(z_{1}(t)\right)+(-1)^{m} \operatorname{Re} \zeta\left(z_{m+1}(t)\right)+\operatorname{Re} q,
\end{aligned}
$$

in directions of end domains, whereas in the other directions

$$
\int_{g\left(z_{k}(t), z_{j}(t)\right)}|d \zeta|=(-1)^{k-1} \operatorname{Re} \zeta\left(z_{k}(t)\right)+(-1)^{j-1} \operatorname{Re} \zeta\left(z_{j}(t)\right)+h_{j k}+o(1) .
$$


Proof. The idea is to use the connection domain $D$ and Lemma (2.3) to construct the geodesic $g(t)$ explicitly. The Lemma (4.11) is then easily verified in each case.

Suppose first that $H_{j}, H_{k}$ are end connected and $k=(j+1)$ for $1 \leqq j \leqq m$. For definiteness we assume that $\zeta\left(E_{j}\right)$ is an upper half plane and $\zeta\left(E_{j}\right)$ is sensed left to right as we move from $H_{j+1}$ into $H_{j}$. Let $z_{1}, z_{2}$ be the farthest zeros left, right on $\partial E_{j}$, respectively. Then set

$$
\begin{aligned}
& G_{1}=\left\{\pi \leqq \arg \left(\zeta-\zeta\left(z_{1}\right)\right) \leqq \pi+\varepsilon\right\}, \\
& G_{2}=\left\{0 \geqq \arg \left(\zeta-\zeta\left(z_{2}\right)\right) \geqq \varepsilon\right\},
\end{aligned}
$$

where $\pi+\varepsilon$ is smaller than the minimum of the two $\zeta$-angles on the side of $E_{j}$ at $z_{1}, z_{2}$. Then set

$$
D_{\varepsilon}=\operatorname{cl} E_{j} \cup G_{1} \cup G_{2} .
$$

For $t \geqq t_{0}, z_{j}(t), z_{j+1}(t) \in D_{\varepsilon}$. It is now clear from (3.11) Figure 1 and Lemma (2.3) that the geodesic $g(t)$ consists of 1,2 or 3 geodesics whose interiors are critical point free. If $\omega$ is the conformal map on $D_{j, j+1}$ then

$$
\int_{g(t)}|d \zeta| \geqq(-1)^{j-1} \operatorname{Re} \omega\left(z_{j}(t)\right)+(-1)^{j} \operatorname{Re} \omega\left(z_{j+1}(t)\right) .
$$

If $1 \leqq j \leqq m$ then $\omega=\zeta$ and

$$
h_{j, j+1}=0 \text {. }
$$

If $j=m$ then $\omega=\zeta$ in $H_{m+1}$ continues analytically through $E_{m+1}$ to the branch $\omega(z)=\zeta\left(e^{2 \pi i} z\right)$ in $H_{1}$, and hence by $(4.5)$ we have

$$
h_{1, m+1}=\operatorname{Re} q \text {. }
$$

Next suppose that $H_{j}, H_{k}$ are strip connected. Suppose first that there is a horizontal connecting strip, critical point free, of maximum width

$$
S=\left\{\left|\operatorname{Im}\left(\zeta-\zeta_{0}\right)\right|<w / 2\right\}
$$

which connects $H_{j}$ to $H_{k}$. That is, $S$ is a strip domain in the global trajectory structure of $d \zeta^{2}$. Let us sense $S$ left to right from $H_{j}$ into $H_{k}$ and let $\omega$ be the analytic continuation of $\zeta$ in $H_{k}$ into $H_{j}$ through $S$. It makes sense to speak of the upper and lower boundaries $L_{1}, L_{2}$ of $S$. Let $z_{1}, z_{2}$ be the first and last zeros on $L_{1}$, let $z_{3}, z_{4}$ be the first and last zeros $L_{2}$. Define 4 angular domains $G_{1}, \ldots, G_{4}$ at these points similar to the domains $G_{1}, G_{2}$ in the last case and set $D_{\varepsilon}=\operatorname{cl} S \cup \bigcup_{1}^{4} G_{v}$. Then again for $t \geqq t_{0}, z_{j}(t), z_{k}(t) \in D_{\varepsilon}$, and from (3.11) Figure 2a and Lemma (2.3), it is clear that $g(t)$ consists of at most 3 geodesics. The outside geodesics have critical point free interiors. The middle horizontal geodesic is independent of $t$. Consequently we have

$$
\int_{g(t)}|d \zeta|=(-1)^{k-1}\left[\operatorname{Re} \zeta\left(z_{k}(t)\right)-\operatorname{Re} \omega\left(z_{j}(t)\right)\right]+o(1) .
$$


But (4.2) implies that $\omega= \pm \zeta+d$ in $H_{j}$ and the fact that $(-1)^{k-1} \operatorname{Re} \omega\left(z_{j}(t)\right)<0$ will tell us the sign. Hence (4.11) holds in this case and

$$
h_{j k}=-\operatorname{Re} d .
$$

If on the other hand $H_{j}, H_{k}$ are strip connected by

$$
S=\left\{\left|\operatorname{Im} e^{-i \theta}\left(\zeta-\zeta_{0}\right)\right|<w / 2\right\}
$$

but not by a horizontal strip, then we know that $0<|\theta|<\pi / 2$ and for definiteness we assume $\theta>0$. We may also assume that $S$ is sensed as before and $L_{1}, L_{2}, \omega$ are defined as before. We want to define new points instead of $z_{1}, z_{2}, z_{3}, z_{4}$. The deformations of $L_{1}, L_{2}$ form a lower and upper boundary of the connecting domain $D_{j k}$, say $L$ and $U$ (see (3.11) Figure 2b). Let $z_{4}$ be the farthest point right on $L$ for which $\sup _{L} \operatorname{Im} \zeta(z)$ is attained and $z_{1}$ the farthest point left on $U$ for which $\inf _{U} \operatorname{Im} \zeta(z)$ is attained. Evidently, no horizontal connecting strips implies

$$
\operatorname{Im} \zeta\left(z_{1}\right) \leqq \operatorname{Im} \zeta\left(z_{4}\right) .
$$

If $\operatorname{Im} \zeta\left(z_{1}\right)=\operatorname{Im} \zeta\left(z_{4}\right)$ then we define $z_{2}$ as the farthest point right on $U$ for which $\operatorname{Im} \zeta\left(z_{1}\right)=\operatorname{Im} \zeta\left(z_{2}\right)$ and $z_{3}$ as the farthest point left on $L$ for which $\operatorname{Im} \zeta\left(z_{3}\right)$ $=\operatorname{Im} \zeta\left(z_{4}\right)$. The previous argument for horizontal connecting strips is then repeated.

If $\operatorname{Im} \zeta\left(z_{1}\right)<\operatorname{Im} \zeta\left(z_{4}\right)$ then $z_{1}$ and $z_{4}$ are called the contact points. Evidently the angular domains

$$
\begin{aligned}
& G_{1}=\left\{\left|\arg \left(\omega-\omega\left(z_{4}\right)\right)\right| \leqq \varepsilon\right\}, \\
& G_{2}=\left\{\left|\arg \left(\omega-\omega\left(z_{1}\right)\right)+\pi\right| \leqq \varepsilon\right\}
\end{aligned}
$$

are critical point free for $\varepsilon$ small enough and the domain

$$
D_{\varepsilon}=G_{1} \cup g\left(z_{1}, z_{4}\right) \cup G_{4}
$$

contains $z_{j}(t), z_{k}(t)$ for $t \geqq t_{0}$. Hence by Lemma (2.3) we have

$$
g\left(z_{j}(t), z_{k}(t)\right)=g\left(z_{j}(t), z_{1}\right)+g\left(z_{1}, z_{4}\right)+g\left(z_{4}, z_{k}(t)\right) .
$$

We approximate the lengths of the first and last geodesics as before to obtain

$$
\begin{aligned}
& \int_{g(t)}|d \zeta| \\
& \quad=(-1)^{k-1}\left[\operatorname{Re} \zeta\left(z_{k}(t)\right)-\operatorname{Re} \zeta\left(z_{4}\right)-\operatorname{Re} \omega\left(z_{j}(t)\right)+\operatorname{Re} \omega\left(z_{1}\right)\right]+\int_{g\left(z_{1}, z_{4}\right)}|d \zeta|+o(1) .
\end{aligned}
$$

Again $\omega= \pm \zeta+d$ in $H_{j}$ and $(-1)^{k-1} \operatorname{Re} \omega\left(z_{j}(t)\right)<0$ implies that $\omega=(-1)^{j-1} \zeta+d$ in $H_{j}$. Hence we find that (4.11) holds and

$$
h_{j k}=(-1)^{k} \operatorname{Re} \zeta\left(z_{4}\right)+(-1)^{j} \operatorname{Re} \zeta\left(z_{1}\right)+\int_{g\left(z_{1}, z_{4}\right)}|d \zeta| .
$$


Finally let us consider the last case that $H_{j}$ is line connected to $H_{k}$. Let $g$ be a connecting geodesic of infinite length in both directions sensed left to right from $H_{j}$ to $H_{k}$. Let $U, L$ denote the deformations obtained by bending the ends of $g$ toward the vertical. In this case $U \cap L=g\left(z_{1}, z_{2}\right)$, where $z_{1}$ is left of $z_{2}$. Clearly $\zeta$ may be defined conformally on both components of the interior of the connecting domain $D_{k l}$ by direct analytic continuations. Let $w_{1}, \ldots, w_{\mu}=z_{1}$ be the zeros on $U$ left of $z_{1}$ and $v_{1}, \ldots, v_{v}=z_{1}$ the zeros on $L$ left of $z_{1}$. For definiteness assume $\operatorname{Im} \zeta$ increases, decreases as we move left on the left vertical end of $U, L$ respectively.

As we move left on the initial segments $g\left(w_{\mu-1}, w_{\mu}\right), g\left(v_{v-1}, v_{v}\right)$ either we move up on the first or we move down on the second since they have disjoint interiors. For definiteness we assume we move down on $g\left(v_{v-1}, v_{v}\right)$. Then let $z_{j k}$ be the farthest point right on $U$, but not right of $z_{1}$ on which $\inf \operatorname{Im} \zeta(z)$ is obtained for points $z \in U$ left of $z_{1}$. We call $z_{j k}$ the contact point of $H_{j}$ to $H_{k}$. Let $w_{j k}$ be the farthest point left on $U$ for which this infimum is obtained. Evidently $w_{j k}=z_{j k}$ unless there are some horizontal geodesics left of $z_{j k}$ on $U$. Similarly there must be a contact point $z_{k j}$ at $H_{k}$ and a corresponding $w_{k j}$. Now we form 4 angular domains $G_{1}, G_{2}, G_{3}, G_{4}$ from $z_{j k}, w_{j k}, z_{k j}, w_{k j}$ as before and set

$$
D_{\varepsilon}=g\left(z_{j k}, z_{k j}\right) \cup \bigcup_{1}^{4} G_{v} .
$$

Clearly for $t \geqq t_{0}, z_{j}(t), z_{k}(t) \in D_{\varepsilon}$ and $g\left(z_{j}(t), z_{k}(t)\right)$ can be written as 5 geodesics

$$
g(t)=g\left(z_{j}(t), w_{j k}\right)+g\left(w_{j k}, z_{j k}\right)+g\left(z_{j k}, z_{k j}\right)+g\left(z_{k j}, w_{k j}\right)+g\left(w_{k j}, z_{k}(t)\right) .
$$

The first and last can be estimated asymptotically as $t \rightarrow \infty$ and we observe as before that (4.11) holds with the formula

$$
h_{j k}=(-1)^{j} \operatorname{Re} \zeta\left(z_{j k}\right)+(-1)^{k} \operatorname{Re} \zeta\left(z_{k j}\right)+\int_{g\left(z_{j k}, z_{k j}\right)}|d \zeta| .
$$

Hence (4.11) holds in all cases. As a corollary we observe that the proof gives us a way to compute the hdac $h_{j k}$. In addition the following interesting relation is clear from the proof.

Corollary (4.17). If $\omega=\zeta+d$ then $h_{j k}(\omega)=h_{j k}(\zeta)+\left((-1)^{k+1}+(-1)^{j+1}\right) \operatorname{Re} d$.

(d) In $\S 4(b)$ we defined $\zeta$ on $U=\{|z|>r\}-V$ and then $\gamma_{r}=\gamma_{1}+\gamma_{2}$ where $\gamma_{2} \subset V$. Now $V$ was chosen as a vertical geodesic so that the horizontal direction adjustment constant $h_{1 k}$ would be well defined. However, for our proof it will be necessary or at least easier if we take $V$ to be a horizontal geodesic. The $a_{k}, b_{k}$ of (4.1) are the same as before. Define $J_{r}$ to be the domain bounded by $\gamma_{r}$ which does not contain $\infty$. Since we want to estimate $\iint_{J_{r}}|d \zeta|^{2}$ asymptotically the following relation is useful.

LEMMA (4.18).

$$
2\left(\sum_{1}^{m-2} w_{j}-\sum_{1}^{m+1} b_{k}\right)=\operatorname{Im} q
$$


We recall that $b_{k}$ is defined in (4.1), $w_{j}$ is the width of the $j$ th strip domain and $q$ is the quasi-period (4.6). To prove it we observe from (4.5), (4.7) that

$$
\int_{\gamma_{r}}|d \operatorname{Im} \zeta|=2(m+1) r+\operatorname{Im} q+o(1)
$$

In strip domains $S_{j}$ and end domains $E_{k}$,

$$
\int_{\gamma_{r} \cap E_{k}}|d \operatorname{Im} \zeta|=2\left(r-b_{k}\right), \quad \int_{S_{j} \cap \gamma_{r}}|d \operatorname{Im} \zeta|=2 w_{j} .
$$

Hence if we add these we obtain

$$
\int_{\gamma_{r}}|d \operatorname{Im} \zeta|=2(m+1) r+2\left(\sum_{1}^{m-2} w_{j}-\sum_{1}^{m+1} b_{k}\right)
$$

and consequently comparison of (4.19) with (4.20) yields (4.18).

We underline the fact that the choice of $\zeta$ is fixed throughout this discussion. However, it is interesting to note that although the values $b_{k}, q$ depend on the choice of $\zeta$, the values $2 \sum_{1}^{m+1} b_{k}+\operatorname{Im} q$ does not.

LEMMA (4.21).

$$
\iint_{J_{r}}|d \zeta|^{2}=\frac{(m+1) \pi}{2} r^{2}+r \operatorname{Im} q+\sum_{1}^{m-2} h_{j} w_{j}+o(1),
$$

where $h_{j}$ is the hdac of the directions defined by the jth strip domain and $w_{j}$ is its width.

Proof. Let $\{\Delta\}$ denote the end and strip domain in the global trajectory structure of $d \zeta^{2}$. Define $\Delta_{r}=\Delta \cap J_{r}$ and notice that

$$
\iint_{J_{r}}|d \zeta|^{2}=\sum_{\Delta} \iint_{\Delta_{r}}|d \zeta|^{2}
$$

Clearly we have

$$
\begin{aligned}
& \iint_{E_{k}}|d \zeta|^{2}=\frac{\pi}{2} r^{2}-2 r b_{k}+O(1), \\
& \iint_{S_{j}}|d \zeta|^{2}=\left(2 r+h_{j}\right) w_{j}+o(1) .
\end{aligned}
$$

Adding these we have

$$
\iint_{J_{r}}|d \zeta|^{2}=(m+1) \frac{\pi}{2} r^{2}+2\left(\sum_{1}^{m-2} w_{j}-\sum_{1}^{m+1} b_{k}\right) r+\sum_{1}^{m-2} h_{j} w_{j}+o(1) .
$$

Now (4.18) implies (4.21). 
5. The extended inequality. (a) The inequalities of Teichmüller and Jenkins represent the limit of the difference between the estimates (above and below) of the change in area of $D_{r}=D \cap J_{r}$ (measured by the density $|d \zeta|^{2}$ ) under the mapping by $h$. The estimates are made by the length-area technique. In this section we shall describe a procedure for overcoming the difficulty in applying the lengtharea technique when $h$ does not have a coefficient normalization.

Let $\left(d \zeta^{2}, R ; h, D\right)$ be a given situation in Teichmüller's case and let $\zeta$ be a fixed branch of the $Q$-integral on $U=\{|z|>r\}-V$, for a horizontal geodesic $V$ on the right end of the boundary of $E_{1}$. Let $\omega$ be any branch of the $Q$-integral defined on $h(U)$. The canonical curves $\gamma_{r}=\gamma(r ; \zeta)$ and exhaustions $J_{r}, D_{r}, \Delta_{r}$ are then defined. We have by (4.3), (3.2) the following expansions:

$$
\begin{aligned}
& \zeta(z)=\sum_{n \geqq 0}^{\prime} \sigma_{n} z^{(m+1) / 2-n}+\sigma_{l} \log z+\sigma_{c}, \\
& \omega(w)=\sum_{n \geqq 0} \lambda_{n} w^{(m+1) / 2-n}+\lambda_{l} \log w+\lambda_{c}, \\
& w=h(z)=z+\sum_{n=0}^{\infty} c_{n} z^{-n} \quad\left(c_{0}=0\right) .
\end{aligned}
$$

In this case $\lambda_{n}=\sigma_{n}, \lambda_{l}=\sigma_{l}$. Then we have the expansion for the composition

$$
\omega(h(z))=\sum_{n \geqq 0}^{\prime} \tau_{n} z^{(m+1) / 2-n}+\dot{\tau}_{l} \log z+\tau_{c}
$$

where $\lambda_{l}=\tau_{l}$ and

$$
\tau_{n}=\sum_{\nu=0}^{n} \lambda_{\nu} c_{n-\nu}^{((m+1) / 2-v)}
$$

with the following conventions. First when $m$ is odd $\lambda_{(m+1) / 2}=\lambda_{l}$. Second

$$
\begin{array}{rlrl}
{[h(z)]^{\alpha}} & =\sum_{k=0}^{\infty} c_{k}^{(\alpha)} z^{\alpha-k} & & (\alpha \neq 0), \\
\lambda_{c}+\log h(z) & =\log z+\sum_{k=0}^{\infty} c_{k}^{(0)} z^{\alpha-k} & (\alpha=0) .
\end{array}
$$

Note that $c_{0}^{(\alpha)}=1$ if $\alpha \neq 0$. For $n \geqq 1$ we have the following formula for $c_{n}^{(\alpha)}$ :

$$
c_{n}^{(\alpha)}=\sum_{1 k_{1}+\cdots+n k_{n}=n ; k_{v} \geqq 0}\left[\left(\begin{array}{c}
\alpha \\
k_{1}+k_{2}+\cdots+k_{n}
\end{array}\right) \prod_{\nu=1}^{n}\left(c_{\nu-1}\right)^{k_{v}}\right]
$$

where

$$
\begin{aligned}
\left(\begin{array}{l}
\alpha \\
n
\end{array}\right) & =\frac{\alpha(\alpha-1) \cdots(\alpha-n+1)}{n !} & & \text { if } \alpha \text { is not a nonnegative integer, } \\
& =\frac{(-1)^{n-1}}{n} & & \text { if } \alpha=0, \\
& =\frac{\alpha !}{n !(\alpha-n) !} & & \text { if } \alpha \text { pos. int., } n \leqq \alpha, \\
& =0 & & \text { if } \alpha \text { pos. int., } n>\alpha,
\end{aligned}
$$


for $n \geqq 1$. It is clear from these formulas that if $\lambda_{n}=\sigma_{n}$ and Jenkins' normalization (3.5) holds then $\tau_{n}=\sigma_{n}$ for $n \leqq[m / 2]$ and $\sigma_{l}=\tau_{l}$. Therefore $H=\zeta \circ h \circ \zeta^{-1}$ on $\zeta(U)$ will have the following normalized expansion:

$$
H(\zeta)=\zeta+\sum_{0 \geqq i \geqq-1} \mu_{t} \zeta^{t}+o\left(|\zeta|^{-1}\right)
$$

where either $t=0$ or $t=1-2 n /(m+1)$ for $(m+1) / 2 \leqq n \leqq m+1$. We remark that the actual order of the remainder term is $O\left(|\zeta|^{-(m+3)(m+1)}\right)$ when $m$ is even and $O\left(|\zeta|^{-(m+3) /(m+1)} \log |\zeta|\right)$ when $m$ is odd. Both of these are $o\left(|\zeta|^{-1}\right)$.

The key observation is that Jenkins' proof depends on the normalization (5.8) of $H$ rather than the normalization (3.5) of $h$. Let us expand on this. Jenkins' result is obtained by estimating the difference in the $Q$-densities of $h\left(D_{r}\right)$ and $D_{r}$. That is,

$$
l(r) \leqq \iint_{h\left(D_{r}\right)}|d \zeta|^{2}-\iint_{D_{r}}|d \zeta|^{2} \leqq u(r) .
$$

The estimates $u(r), l(r)$ are in terms of the coefficients of $H$. They are obtained by using essentially Schwarz's inequality to compare length to area. If they are so precise that

$$
u(r)-l(r)=A+o(1)
$$

then the result $A \geqq 0$ can be concluded. Expansion (5.8) of $H$ and Jenkins' technique of estimation gives (5.9). However, if fewer initial coefficients of $h$ are nonzero than in Jenkins' normalization (3.5) then $H$ will have some nonzero coefficients on its positive fractional terms and

$$
u(r)-l(r)=K\left|\mu_{t_{0}}\right|^{2} r^{2 t}+o\left(r^{2 t}\right) .
$$

The result $A \geqq 0$ is not obtained since the difference diverges.

(b) To overcome this obstacle we change the point of view. First the estimates are essentially on $\iint_{h\left(D_{r}\right)}|d \zeta|^{2}$ rather than the difference. (Estimation of the difference merely simplifies computation.) Hence we estimate the area of $h\left(D_{r}\right)$. Second, we choose a new density $|d \omega|^{2}$ of the same type as $|d \zeta|^{2}$ and estimate the $|d \omega|^{2}$ density of $h\left(D_{r}\right)$. The choice of $d \omega^{2}$ is made so that when a branch of its integral $\omega$ is used to form $H=\omega \circ h \circ \zeta^{-1}$, then $H$ will have the expansion (5.8) and the lengtharea estimates will give (5.9).

(c) The actual normalization we need will require the additional restriction Im $\mu_{0}=0$ when $m+1$ is odd. We shall call $\left(d \omega^{2}, \omega\right)$ an allowable pair if $d \omega^{2}$ is of the form (3.1) and $H=\omega \circ h \circ \zeta^{-1}$ has expansion (5.8) with the additional restriction. We now prove the existence of allowable pairs.

Lemma (5.11). For each situation $\left(d \zeta^{2}, R ; h, D\right)$ and choice of $\zeta$ in Teichmüller's case there is a space of allowable pairs $\left(d \omega^{2}, \omega\right)$ which can be parametrized by $m-1$ real variables.

Proof. We consider $d \omega^{2}=P(w) d w^{2}$ of form (3.1). Then $\omega(h(z))$ has expansion (5.4) where the coefficients are given by formulas (5.6), (5.7) and (4.4). We compare this to the expansion (5.1) for $\zeta(z)$ Then $\omega \circ h \circ \zeta^{-1}=H$ will have the desired 
expansion if and only if

$$
\begin{aligned}
\sigma_{n} & =\tau_{n} & & (\text { for } 0 \leqq n \leqq[m / 2]), \\
\sigma_{l} & =\tau_{l} & & \text { (for } m \text { odd), } \\
\operatorname{Im} \sigma_{c} & =\operatorname{Im} \tau_{c} & & \text { (for } m \text { even). }
\end{aligned}
$$

Now let $d \omega^{2}=P(w) d w^{2}=\gamma w^{m-1}\left(1+\sum_{v=1}^{m-1} \delta_{v} w^{-v}\right) d w^{2}$. Then $\sigma_{0}=\tau_{0}$ if and only if $\alpha=\gamma$. By formulas (4.4) and (5.5) it is clear that

$$
\tau_{n}=K \alpha^{1 / 2} \delta_{n}+G\left(\alpha, \delta_{1}, \ldots, \delta_{n-1} ; h\right)
$$

Since we have $\alpha \neq 0$ it is clear that (5.12) holds for a unique choice of $\gamma$ and $\delta_{1}, \ldots, \delta_{[(m+1) / 2]}$ and, in the even case, the requirement $\operatorname{Im} \sigma_{c}=\operatorname{Im} \tau_{c}$. Hence $m+3$ real variables are required. Counting the constant of integration there are $2(m+1)$ real variables available, hence $2(m+1)-(m+3)=(m-1)$ remain free to parametrize the space $\left\{\left(d \omega^{2}, \omega\right)\right\}$.

COROLlaRY (5.13). If $\left(d \omega^{2}, \omega\right)$ is allowable then

(i) $d \omega^{2}$ has the same horizontal directions $\theta_{k}$ as $d \zeta^{2}$.

(ii) $\omega$ has the same quasi-period as $\zeta$ if $(m+1)$ is even and the same imaginary part of its quasi-period as $\zeta$ if $m+1$ is odd.

This follows from (5.12).

(d) We now state our main result.

Theorem (5.14) (COMPlete Teichmüller Inequalities). For each situation $\left(d \zeta^{2}, R ; h, D\right)$ in Teichmüller's case and choice of $\zeta$ let $\left(d \omega^{2}, \omega\right)$ be allowable. Then

$$
\begin{aligned}
(m+1) \pi \operatorname{Re} \mu_{-1}+\operatorname{Im}\left(\mu_{0} q\right)+2 & \sum_{1}^{m-2} A_{j}(\omega) c_{j}(\zeta) \\
& \leqq \sum_{1}^{m-2} A_{j}(\zeta) c_{j}(\zeta)+\sum_{1}^{m-2} B_{j}(\omega) d_{j}(\omega),
\end{aligned}
$$

where the constants in (5.15) are listed in the Table of Constants (5.16).

Equality occurs if and only if $h$ is a horizontal isometry, in other words if and only if $h=\omega^{-1} \circ \zeta$ on an open subset of $D$.

(5.16) Table of Constants.

$\mu_{0}, \mu_{-1}$ are the constants in the expansion (5.8) of $H=\omega \circ f \circ \zeta^{-1}$.

$q$ is the quasi-period of $\omega$.

$c_{j}(\zeta), d_{j}(\omega)$ are the widths of the strip domain in the global trajectory structure of $d \zeta^{2}, d \omega^{2}$ respectively.

$A_{j}(\zeta), B_{j}(\omega)$ are the hdac (4.9) of $\zeta, \omega$ corresponding to the directions of the $j$ th strip domain of $d \zeta^{2}, d \omega^{2}$ respectively.

$A_{j}(\omega)$ are the $\omega$-hdac (4.9) of the directions corresponding to the $j$ th strip domain of $d \zeta^{2}$.

In Teichmüller's and Jenkins' case $\left(d \zeta^{2}, \zeta\right)$ itself is allowable and hence $A_{j}(\omega)$ $=B_{j}(\zeta)=A_{j}(\zeta), c_{j}(\zeta)=d_{j}(\omega)$. In Teichmüller's case $\mu_{0}=0$ too. Hence Teichmüller's Theorem is

$$
(m+1) \pi \operatorname{Re} \mu_{-1} \leqq 0
$$


and Jenkins' Theorem is

$$
(m+1) \pi \operatorname{Re} \mu_{-1}+\operatorname{Im}\left(\mu_{0} q\right) \leqq 0 .
$$

The proof of Theorem (5.14) is carried out in the remainder of this paper.

6. The upper estimate $u(r)$. We have $\zeta$ defined on $U=\{|z|>r\}-V$, where $V$ is a horizontal geodesic emanating from $\infty$ on the right side of the boundary of $E_{1}$. The canonical curves $\gamma=\gamma_{r}=\gamma_{r}(\zeta)$ and exhaustions $J_{r}, D_{r}, \Delta_{r}$ are then defined. We wish to estimate $\iint_{h\left(D_{r}\right)}|d \omega|^{2}$ from above.

The complement of $h\left(D_{r}\right)$ is a compact set $K$ of finite $|d \omega|^{2}$-density. Since $J_{h(\gamma)}$ is a disjoint union of $h\left(D_{r}\right)$ and $K$ we may write

$$
\iint_{h\left(D_{r}\right)}|d \omega|^{2} \leqq \iint_{J_{h(\gamma)}}|d \omega|^{2} .
$$

Equality occurs if and only if the $|d \omega|^{2}$-density of $K$ is zero. That is equivalent to $K$ having Lebesgue density zero. Our estimate $u(r)$ will be an asymptotic expansion of $\iint_{J_{h(\gamma)}}|d \omega|^{2}$ in terms of our constants. To that end we set $B=\gamma_{r}(\omega)$ and write

$$
\iint_{J_{h(\gamma)}}|d \omega|^{2}=\iint_{J_{B}}|d \omega|^{2}+\left(\iint_{J_{h(\gamma)}}|d \omega|^{2}-\iint_{J_{B}}|d \omega|^{2}\right) .
$$

Lemma (4.21) tells us that

$$
\iint_{J_{B}}|d \omega|^{2}=\frac{m+1}{2} \pi r^{2}+r \operatorname{Im} q+\sum_{j=1}^{m-2} B_{j}(\omega) d_{j}(\omega)+o(1),
$$

where $\operatorname{Im} q$ is the common imaginary part of the quasi-periods of $\zeta$ and $\omega$, and $B_{j}(\omega), d_{j}(\omega)$ are defined in the Table of Constants (5.16).

The task remains to estimate the difference in the $|d \omega|^{2}$-densities of $J_{h(\gamma)}$ and $J_{B}$. This will be accomplished by the use of Stokes' Theorem. Since $\omega$ is not defined on these regions we must consider subregions of equal density, where $\omega$ can be defined. Technically this is accomplished in the paragraph below. Diagram (6.4) may be used to help keep account of the arcs in the ensuing discussion.

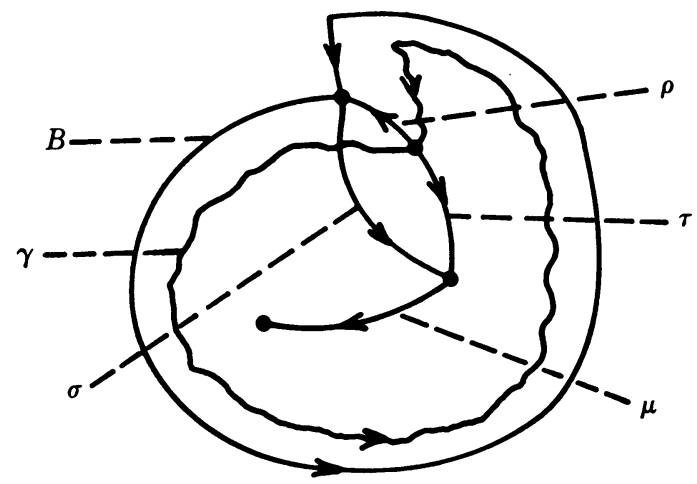


Let $\mu=\mu(t)(0 \leqq t \leqq 1)$ denote a smooth Jordan arc in $R-\{\infty\}$ which contains all the zeros of $d \omega^{2}$. For $r \geqq r_{0}, \mu$ will be contained in a Euclidean disk whose closure lies in $J_{B} \cap J_{h(\gamma)}$. We may assume that $r_{0}$ is so large that $h(\gamma(0)), B(0)$ lie in a disk whose closure is disjoint from the first disk. Let $\rho=\rho(t)(0 \leqq t \leqq 1)$ be a smooth Jordan arc which runs from $h(\gamma(0))$ to $B(0)$ in this disk. Let $\sigma=\sigma(t)(0 \leqq t \leqq 1)$ be a smooth Jordan arc in $J_{B}-\mu$ which runs from $B(0)$ to $\mu(0)$. Finally let $\tau=\tau(t)$ $(0 \leqq t \leqq 1)$ be a smooth Jordan arc in $J_{h(\gamma)}-\mu$ which runs from $h(\gamma(0))$ to $\mu(0)$ and which is homotopic to $\rho \sigma$ in $R-\{\infty\}-\mu$.

Since the supports of these $\operatorname{arcs} \mu, \sigma, \tau, \rho$ have zero $|d \omega|^{2}$-density we may write

$$
\iint_{J_{h(\gamma)}}|d \omega|^{2}-\iint_{J_{B}}|d \omega|^{2}=\iint_{J_{h(\gamma)}-\tau \mu}|d \omega|^{2}-\iint_{J_{B}-\sigma \mu}|d \omega|^{2} .
$$

But $J_{h(\gamma)}-\tau \mu$ and $J_{B}-\sigma \mu$ are simply connected, critical point free domains. Consequently the branch of $\omega$ on the intersection of $h(U)$ and the disk containing $B(0)$, $h(\gamma(0))$ may be continued analytically into $J_{h(\gamma)-\tau \mu}, J_{B-\sigma \mu}$ to obtain analytic functions $\omega_{2}, \omega_{1}$ respectively on these regions. They have continuous extensions to the sides $\rho_{j}, \sigma_{j}, \tau_{j}, \mu_{j}$ (for $\left.j=l, r\right)$. Therefore by Stokes' Theorem we have

$$
\iint_{J_{h(\gamma)}-\tau \mu}|d \omega|^{2}-\iint_{J_{B}-\sigma \mu}|d \omega|^{2}=\operatorname{Re}\left(\frac{1}{2 i} \int_{C_{2}} \bar{\omega}_{2} d \omega_{2}-\frac{1}{2 i} \int_{C_{1}} \bar{\omega}_{1} d \omega_{1}\right),
$$

where

$$
\begin{aligned}
& C_{1}=B_{1}+B_{2}+\sigma_{l}+\mu_{l}-\mu_{r}-\sigma_{r}, \\
& C_{2}=h\left(\gamma_{1}\right)+h\left(\gamma_{2}\right)+\tau_{l}+\mu_{l}-\mu_{r}-\tau_{r} .
\end{aligned}
$$

Consequently, in view of the homotopy between $\tau$ and $\rho \sigma$, the quasi-periodicity (4.5) of $\omega$, and cancellation of the integrals over $\mu_{r}, \mu_{l}$, we may write

$$
\begin{aligned}
\operatorname{Re} & \frac{1}{2 i}\left(\int_{C_{2}} \bar{\omega}_{2} d \omega_{2}-\int_{C_{1}} \bar{\omega}_{1} d \omega_{1}\right) \\
\quad & =\operatorname{Re} \frac{1}{2 i} \int_{\rho_{r}-\rho_{l}} \bar{\omega} d \omega+\operatorname{Re} \frac{1}{2 i} \int_{h\left(\gamma_{1}\right)-B_{1}} \bar{\omega} d \omega+\operatorname{Re} \frac{1}{2 i} \int_{h\left(\gamma_{2}\right)-B_{2}} \bar{\omega} d \omega .
\end{aligned}
$$

We must keep in mind which branch of $\omega$ is defined on these various arcs. We do this by saying that $\rho_{l}$ connects $h\left(\gamma_{1}(0)\right)$ to $B_{1}(0)$ and $\rho_{r}$ connects $h\left(\gamma_{2}(1)\right)$ to $B_{2}(1)$. To estimate the first of these 3 integrals we use (4.5), the quasi-periodicity of $\omega$ to write

$$
\begin{aligned}
\operatorname{Re} \frac{1}{2 i} \int_{\rho_{r}-\rho_{l}} \bar{\omega} d \omega & =\operatorname{Re} \frac{1}{2 i} \int_{\rho_{l}}\left(\overline{\varepsilon\left(\omega^{+} q\right)} \varepsilon d \omega-\bar{\omega} d \omega\right) \\
& =\operatorname{Re} \frac{1}{2 i} \bar{q} \int_{\rho_{l}} d \omega=\operatorname{Re}\left[\frac{1}{2 i} \bar{q}(\omega(B(0))-\omega(h(\gamma(0))))\right],
\end{aligned}
$$

where $\varepsilon=(-1)^{m+1}$. But $\omega(B(0))=r, \omega(h(\gamma(0)))=H(r)=r+\mu_{0}+o(1)$ and consequently $-\operatorname{Re}(1 / 2 i) \bar{q} \mu_{0}=(1 / 2) \operatorname{Im} q \bar{\mu}_{0}$ implies

$$
\operatorname{Re} \frac{1}{2 i} \int_{\rho_{r}-\rho_{l}} \bar{\omega} d \omega=\frac{1}{2} \operatorname{Im}\left(q \bar{\mu}_{0}\right)+o(1) .
$$


To estimate the second of these 3 integrals in (6.7) recall that $\omega\left(B_{1}(\theta)\right)=r e^{i \theta}$ for $\theta_{1} \leqq \theta \leqq \theta_{2}$. Therefore

$$
\left.\left.\operatorname{Re} \frac{1}{2 i} \int_{h\left(\gamma_{1}\right)-B_{1}} \bar{\omega} d \omega=\operatorname{Re} \frac{1}{2 i} \int_{\theta_{1}}^{\theta_{2}} \overline{\left(H \left(r e^{i \theta}\right.\right.}\right) d H\left(r e^{i \theta}\right)-\overline{r e^{i \theta}} d r e^{i \theta}\right) .
$$

Now we expand $H\left(r e^{i \theta}\right)$ by (5.8).

$$
H\left(r e^{i \theta}\right)=r e^{i \theta}+\mu_{0}+\sum \mu_{t} r^{t} e^{i t \theta}+o\left(r^{-1}\right),
$$

where sums are indexed by $0>t \geqq-1$ here. Therefore (6.10) becomes

$$
\begin{aligned}
\operatorname{Re} & \frac{1}{2 i} \int_{h\left(\gamma_{1}\right)-B_{1}} \bar{\omega} d \omega \\
& =\operatorname{Re} \frac{1}{2} \int_{\theta_{1}}^{\theta_{2}}\left[\bar{\mu}_{0} r e^{i \theta}+\sum \bar{\mu}_{t} t^{t+1} e^{i(1-t) \theta}+\sum t \mu_{t} r^{t+1} e^{i(t-1) \theta}+o(1)\right] d \theta,
\end{aligned}
$$

where

$$
\begin{aligned}
& \theta_{1}=b_{1} r^{-1}+o\left(r^{-1}\right), \\
& \theta_{2}=(m+1) \pi+\varepsilon b_{1} r^{-1}+\varepsilon \operatorname{Im} q^{r-1}+o\left(r^{-1}\right),
\end{aligned}
$$

where $\varepsilon=(-1)^{m+1}$. Now if $s= \pm(1 \pm t)= \pm 2 n /(m+1) \neq 0, s=1$, or $s=0$, then

$$
\begin{aligned}
\int_{\theta_{1}}^{\theta_{2}} e^{i s \theta} d \theta & =\varepsilon r^{-1} \operatorname{Im} q+o\left(r^{-1}\right) & & \text { if } \delta= \pm 2 n /(m+1) \neq 0 \\
& =(1-\varepsilon) i+\varepsilon r^{-1} \operatorname{Im} q+o\left(r^{-1}\right) & & \text { if } \delta=1 \\
& =(m+1) \pi+\varepsilon r^{-1} \operatorname{Im} q+o\left(r^{-1}\right) & & \text { if } \delta=0 .
\end{aligned}
$$

Hence after integration the two sums in (6.11) are $o(1)$ because $r^{t}=o(1)$. Only the term involving $\mu_{0}$ is significant and hence by (6.12) we have

$$
\operatorname{Re} \frac{1}{2 i} \int_{h\left(\gamma_{1}\right)-B_{1}} \bar{\omega} d \omega=(1+\varepsilon) r \operatorname{Im} \mu_{0} / 2+\varepsilon \operatorname{Re} \mu_{0} \operatorname{Im} q / 2+o(1)
$$

Hence if $m+1$ is odd we are required to impose the condition Im $\mu_{0}=0$ to keep this term from making $u(r)-l(r)$ diverge. Therefore

$$
\operatorname{Re} \frac{1}{2 i} \int_{h\left(\gamma_{1}\right)-B_{1}} \bar{\omega} d \omega=\frac{\varepsilon}{2} \operatorname{Re} \bar{\mu}_{0} \operatorname{Im} q+o(1) .
$$

To estimate the third integral in (6.7) recall that $B_{2} \subset V$, a horizontal geodesic. Note that $\omega\left(B_{2}(t)\right)=r e^{i \theta_{2}}(1-t)+\varepsilon\left(r e^{\theta_{1}}+q\right) t, \omega\left(h\left(\gamma_{2}(t)\right)\right)=H\left(\omega\left(B_{2}(t)\right)\right)=\omega\left(B_{2}(t)\right)$ $+\mu_{0}+o(1)$. So

$$
\begin{aligned}
\operatorname{Re} \frac{1}{2 i} \int_{h\left(\gamma_{2}\right)-B_{2}} \bar{\omega} d \omega & =\operatorname{Re} \frac{1}{2 i} \int_{0}^{1} \bar{\mu}_{0} d \omega\left(B_{2}(t)\right) \\
& =\operatorname{Re}(1 / 2 i) \bar{\mu}_{0}\left(\omega\left(B_{2}(1)\right)-\omega\left(B_{2}(0)\right)\right) \\
& =(\varepsilon / 2) \operatorname{Re} q \operatorname{Im} \bar{\mu}_{0}+o(1) .
\end{aligned}
$$


Addition of (6.9), (6.14), (6.15) gives an expansion in (6.7). Combining this with (6.5) and (6.6) gives

$$
\iint_{J_{h(\gamma)}}|d \omega|^{2}-\iint_{J_{B}}|d \omega|^{2}=\frac{1}{2}(1+\varepsilon) \operatorname{Im}\left(\bar{\mu}_{0} q\right)+o(1) .
$$

Adding (6.3) and (6.15) gives

$$
u(r)=\frac{m+1}{2} \pi r^{2}+r \operatorname{Im} q+\sum_{j=1}^{m-2} B_{j}(\omega) d_{j}(\omega)+\delta \operatorname{Im}\left(\bar{\mu}_{0} q\right),
$$

where $\delta=(1+\varepsilon) / 2, \varepsilon=(-1)^{m+1}$.

7. The lower estimate $l(r)$. To estimate the $|d \omega|^{2}$-density of $h\left(D_{r}\right)$ from below we must first change variables. Consider the $Q$-domains in the global trajectory structure of $d \zeta^{2}$. From them we remove the finite number of unobstructed trajectories which contain the horizontal geodesics of $\partial D$ or the points in $h^{-1}\left(Z\left(d \omega^{2}\right)\right)$. (Recall that $Z\left(d \omega^{2}\right)$ is the set of zeros of $d \omega^{2}$.) We shall continue to call the resulting domains $\{\Delta\}$, end domains $\left\{E_{k}\right\}_{k=1}^{m+1}$, or strip domains $\left\{S_{j}\right\}_{j=1}^{N}$, according to whether $\zeta\left(E_{k}\right)=\left\{(-1)^{k+1} \operatorname{Im} \zeta>b_{k}\right\}$ or $\zeta\left(S_{j}\right)=\left\{\left|\operatorname{Im}\left(\zeta-\zeta_{j}\right)\right|<w_{j} / 2\right\}$. The relation between these new constants $b_{k}$ and $w_{j}$ is the same as before (see Lemma (4.18)). Since the boundaries have $|d \omega|^{2}$-density zero and the domains are disjoint and dense we may write

$$
\iint_{h\left(D_{r}\right)}|d \omega|^{2}=\sum \iint_{\Delta_{r}}|d \omega|^{2} .
$$

On $\Delta$ we may define $\zeta$ conformally; on $h(\Delta) \omega$ is defined analytically. We emphasize that these are not necessarily the same $\zeta$ on $U$ and $\omega$ on $h(U)$ as before. Although $H=\omega \circ h \circ \zeta^{-1}$ on $\zeta(\Delta)$ depends on the choice of the integrals, $\left|H^{\prime}(\zeta)\right|$ does not, because of the nature of the relations (4.2) between the branches. Therefore change variables to obtain

$$
\iint_{\Delta_{r}}|d \omega|^{2}=\iint_{\zeta\left(\Delta_{r}\right)}\left|H^{\prime}(\zeta)\right|^{2} d \xi d \eta
$$

where $\zeta=\xi+i \eta$. Now we use the essence of Schwarz's inequality, $a^{2} \geqq 2 a b-b^{2}$ to write

$$
\iint_{\zeta\left(\Delta_{r}\right)}\left|H^{\prime}(\zeta)\right|^{2} d \xi d \eta \geqq 2 \iint_{\zeta\left(\Delta_{r}\right)}\left|H^{\prime}(\zeta)\right| d \xi d \eta-\iint_{\zeta\left(\Delta_{r}\right)} d \xi d \eta .
$$

Now the sum of the last integrals is the $|d \zeta|^{2}$-density of $J_{r}$, which may be estimated by Lemma (4.21),

$$
\begin{aligned}
\sum_{\Delta_{r}} \iint_{\zeta\left(\Delta_{r}\right)} d \xi d \eta & =\iint_{J_{r}}|d \zeta|^{2} \\
& =\frac{m+1}{2} \pi r^{2}+r \operatorname{Im} q+\sum A_{j}(\zeta) c_{j}(\zeta)+o(1)
\end{aligned}
$$


where $q$ is the quasi-period and $A_{j}(\zeta), c_{j}(\zeta)$ are the constants in the Table of Constants (5.16). Now by Fubini's Theorem

$$
\iint_{\zeta\left(\Delta_{r}\right)}\left|H^{\prime}(\zeta)\right| d \xi d \eta=\int_{b}^{a}\left(\int_{l(\eta)}\left|H^{\prime}(\zeta)\right| d \xi\right) d \eta
$$

But $l(\eta)$ is a $|d \zeta|$ horizontal geodesic and on it $d \xi=|d \zeta|$. Thus

$$
\int_{l(\eta)}\left|H^{\prime}(\zeta)\right| d \xi=\int_{l(\eta)}\left|H^{\prime}(\zeta)\right||d \zeta|=\int_{h(l(\eta))}|d \omega| .
$$

Let $g(\eta)$ denote the $|d \omega|$-geodesic which joins the end points of $h(l(\eta))$. Then

$$
\int_{h(l(\eta))}|d \omega| \geqq \int_{g(\eta)}|d \omega|,
$$

with equality if and only if $h(l(\eta))=g(\eta)$, by the uniqueness of geodesics (3.6). The end points of $g(\eta)$ are values of $h$ taken at points on $\gamma_{r}$. We consider now the original choice of $\omega, \zeta$ for which $H=\omega \circ h \circ \zeta^{-1}$ has expansion (5.8). By Corollary (5.13) and the expansion (5.8) for $H$ it is clear that $h$ preserves the horizontal directions $\theta_{k}$. Therefore if the end points of $\zeta(l, \eta)$ are $r e^{i \theta(\eta)}, r e^{i \psi(\eta)}$ we have in an end domain $E_{k}$, by Lemma (5.11),

$$
\int_{g(\eta)}|d \omega| \geqq(-1)^{k-1} \operatorname{Re} H\left(r e^{i \theta(\eta)}\right)+(-1)^{k} \operatorname{Re} H\left(r e^{i \psi(\eta)}\right),
$$

in a strip domain $S_{j}$, by Lemma (5.11),

$$
\int_{g(\eta)}|d \omega|=(-1)^{k+1} \operatorname{Re} H\left(r e^{i \theta(\eta)}\right)+(-1)^{l+1} \operatorname{Re} H\left(r e^{i \psi(\eta)}\right)+A_{j}(\omega)+o(1),
$$

where $H$ is the standard function on $\zeta(U)$ which has expansion (5.8) (see (5.11)), $\theta_{k}, \theta_{l}$ are the directions of $S_{j}$ and $A_{j}(\omega)$ is the $\omega$-hdac corresponding to the directions $\theta_{k}, \theta_{l}$. Now integration with respect to $\eta$ on the end domains gives

$$
\sum_{k=1}^{m+1} \iint_{\zeta\left(E_{k} \cap J_{r}\right)}\left|H^{\prime}(\zeta)\right| d \xi d \eta \geqq \sum_{k=1}^{m+1} \int_{\zeta\left(E_{k} \cap \gamma_{1}\right)} \operatorname{Re} H(\zeta) d \operatorname{Im} \zeta .
$$

Now in a strip domain we have estimates (7.9) and since the width is independent of $r$ we have, for $c_{j}(\zeta)$ the width of $S_{j}, A_{j}(\omega)$ the hdac of $\omega$ with respect to the directions of $S_{j}$,

$$
\iint_{\zeta\left(S_{j} \cap J_{r}\right)}\left|H^{\prime}(\zeta)\right| d \xi d \eta \geqq \int_{\zeta\left(S_{j} \cap \gamma_{1}\right)} \operatorname{Re} H(\zeta) d \operatorname{Im} \zeta+A_{j}(\omega) c_{j}(\zeta)+o(1) .
$$

Adding (7.10) and (7.11) we obtain

$$
\sum_{\Delta} \iint_{\zeta\left(\Delta_{r}\right)}\left|H^{\prime}(\zeta)\right| d \xi d \eta \geqq \int_{\zeta\left(\gamma_{1}\right)} \operatorname{Re} H(\zeta) d \operatorname{Im} \zeta+\sum_{j=1}^{m-2} A_{j}(\omega) c_{j}(\zeta)+o(1)
$$


Now $H$ has expansion (5.8) and $\gamma_{1}$ has the property $\zeta\left(\gamma_{1}(\theta)\right)=r e^{i \theta}, \theta_{1}(r) \leqq \theta \leqq \theta_{2}(r)$. Therefore

$$
\begin{aligned}
\int_{\gamma_{1}} \operatorname{Re} H(\zeta) d \operatorname{Im} \zeta & \\
=\frac{1}{4} \int_{\theta_{1}}^{\theta_{2}} & \left(r e^{i \theta}+\mu_{0}+\sum \mu_{t} r^{t} e^{i t \theta}+r e^{-i \theta}+\bar{\mu}_{0}+\sum \bar{\mu}_{t} r^{t} e^{-i t \theta}\right) \\
& \times\left(r e^{i \theta}+\sum t \mu_{t} r^{t} e^{i t \theta}+r e^{-i \theta}+\sum t \bar{\mu}_{t} r^{t} e^{-i t \theta}\right) d \theta+o(1) .
\end{aligned}
$$

Now if we multiply these terms, collect in powers of $r$, and integrate, we see by using (6.12) that $(7.13)$ reduces to

$$
\begin{aligned}
\int_{\gamma_{1}} \operatorname{Re} & H(\zeta) d \operatorname{Im} \zeta \\
= & \frac{1}{4} \int_{\theta_{1}}^{\theta_{2}}\left[\left(r^{2} e^{2 i \theta}+2 r^{2}+r^{2} e^{-2 i \theta}\right)\right. \\
& \left.\quad+\left(\mu_{0}+\bar{\mu}_{0}\right)\left(r e^{i \theta}+r e^{-i \theta}\right)+\left(\bar{\mu}_{-1}+\mu_{-1}\right)\right] d \theta+o(1) \\
=((m+1) / 2) \pi r^{2}+r & \operatorname{Im} q+\operatorname{Re} \mu_{0} \operatorname{Im} q+((m+1) / 2) \pi \operatorname{Re} \mu_{-1}+o(1) .
\end{aligned}
$$

If we put (7.14) into (7.12) and then put (7.12) and (7.4) into the sum of the terms (7.3) we obtain

$$
\begin{aligned}
l(r)= & ((m+1) / 2) \pi r^{2}+r \operatorname{Im} q+(m+1) \pi \operatorname{Re} \mu_{-1}+2 \operatorname{Re} \mu_{0} \operatorname{Im} q \\
& +2 \sum_{1}^{m-2} A_{j}(\omega) c_{j}(\zeta)-\sum_{j=1}^{m-2} A_{j}(\zeta) c_{j}(\zeta)+o(1) .
\end{aligned}
$$

8. Equality statement and other remarks. (a) Our inequality (5.15) follows by substituting the expansions (6.16), (7.15) for $u(r), l(r)$ into (5.9). Suppose now that equality holds. Then all inequalities must be equalities. In particular (6.1) implies that the complement $K$ of $h(D)$ has zero $|d \omega|^{2}$-density and since $K$ is bounded from $\infty$, zero Lebesgue density. In the lower estimates the length-area inequality implies $\left|H^{\prime}\right| \equiv 1$, but the inequalities (7.7) and (7.8) must be equality so $H^{\prime}(\zeta) \equiv 1$. Therefore $H(\zeta) \equiv \zeta+d$ and $h=\omega^{-1} \circ \zeta$ for appropriate $\omega$ on an open subset of $D$.

Conversely if $h=\omega^{-1} \circ \zeta$ on $D$ then by analytic continuation it must hold at all but a finite number of points in $D$ for appropriate choice of $\omega$. Clearly the lower inequalities must all be equalities. It remains only to show that the Lebesgue area of $K$ is zero. But $K$ is bounded by geodesics of finite length. If int $K \neq \varnothing$ then $K$ would contain a closed geodesic which is ridiculous.

As a corollary we see that $2 \sum A_{j}(\omega) c_{j}(\zeta)=\sum A_{j}(\zeta) c_{j}(\zeta)+\sum B_{j}(\omega) d_{j}(\omega)$ must also hold when $h=\omega^{-1} \circ \zeta$. Indeed much more must hold. We shall not consider that problem here.

(b) Corollary (4.17) allows an alternate expression for (5.15) which we give here. The upper estimate should be the same $u(r)$. In the lower estimate the estimates on end domains should be the same. We may assume $\mu_{0}$ is zero since these estimates 
are independent of the choice of $\mu_{0}$. On the strip domains $\omega+\mu_{0}$ sends the curve $B_{1}$ into a curve translated by $\pm \operatorname{Re} \mu_{0}$. Indeed the effect is to translate by $(-1)^{k-1} \operatorname{Re} \mu_{0}$ in $H_{k}$. Therefore if $\theta_{k(j)}, \theta_{l(j)}$ are the directions of $S_{j}$ and $\delta_{j}=\frac{1}{2}\left(1+(-1)^{k(j)+l(j)}\right)$ then we have

$$
\begin{aligned}
\iint_{\zeta\left(S_{j} \cap J_{r}\right)}\left|H^{\prime}(\zeta)\right| d \xi d \eta & \geqq 2 r c_{j}(\zeta)+\left(A_{j}(\omega)+2 \operatorname{Re} \mu_{0} \delta_{j}\right) c_{j}(\zeta)+o(1) \\
& =\int_{\zeta\left(\gamma_{1} \cap S_{j}\right)} \operatorname{Re} H(\zeta) d \operatorname{Im} \zeta+A_{j}(\omega) c_{j}(\zeta)+o(1) .
\end{aligned}
$$

Adding this and comparing results both forms of $l(r)$ yield an interesting relation.

Corollary (8.1).

$$
2 \sum_{j=1}^{m-2}\left(\operatorname{Re} \mu_{0}\right) \delta_{j}=\operatorname{Re} \mu_{0} \operatorname{Im} q .
$$

We may substitute this into (7.15) to alter the form of (5.15) if we desire.

(c) We have given the formulas necessary to compute $\mu_{-1}, \mu_{0}$ namely the formulas for $\tau_{n}, \sigma_{n}$. We wish to indicate here how to compute the adjustment constants. The strip constants are

$$
\begin{aligned}
& c_{j}(\zeta)=\operatorname{Im} \int_{a_{j}}^{b_{j}} Q^{1 / 2} d z, \\
& d_{j}(\omega)=\operatorname{Im} \int_{c_{j}}^{d_{j}} P^{1 / 2} d w,
\end{aligned}
$$

where $a_{j}, b_{j}$ and $c_{j}, d_{j}$ are zeros on the boundaries of those strips $S_{j}, T_{j}$ in the global trajectory structures of $d \zeta^{2}, d \omega^{2}$ respectively. The path is any path joining them in the strip.

The hdac were of the form

$$
h_{k j}=\operatorname{Re}\left[(-1)^{k} \zeta\left(z_{k}\right)+(-1)^{l} \zeta\left(z_{l}\right)\right]+g\left(z_{k}, z_{l}\right) .
$$

We indicate an obvious method for finding $g(z, w)$ which we call contraction. Let $\gamma$ denote any arc which joins $z_{k}$ to $w_{k}$. Then set

$$
F(t, s)=g\left(z_{k}, \zeta(s)\right)+\gamma_{s}
$$

where $\gamma_{s}(t)=\gamma(t)$ for $s \leqq t \leqq 1$. Since it is clear how $g\left(z_{k}, \gamma(s)\right)$ changes locally this homotopy gives a canonical way to find $g\left(z_{k}, w_{k}\right)$. It then remains only to compute its length.

\section{BIBLIOGRAPHY}

1. James A. Jenkins, Univalent functions and conformal mapping, 2nd rev. ed., SpringerVerlag, Berlin, 1965.

2. - An addendum to the general coefficient theorem, Trans. Amer. Math. Soc. 107 (1963), 125-128. MR 26 \#5154. 
3. James A. Jenkins, On the global structure of the trajectories of a positive quadratic differential, Illinois J. Math. 4 (1960), 405-412. MR 23 \#A1794.

4. Arthur E. Obrock, An inequality for certain schlicht functions, Proc. Amer. Math. Soc. 17 (1966), 1250-1253. MR 34 \#6075.

5. A. C. Schaeffer and D. C. Spencer, Coefficient regions for schlicht functions, Amer. Math. Soc. Colloq. Publ., vol. 35, Amer. Math. Soc., Providence, R. I., 1950. MR 12, 326.

6. O. Teichmüller, Ungleichungen zwischen den Koeffizienten schlichter Funktionen, S.-B. Preuss. Akad. Wiss. Phys.-Math. K1. 1938, 363-375.

\section{PuRdue UNIVERSity,}

LAFAYETTE, INDIANA 47907 